fatty liver disease. However, data on the use of aminotransferases to monitor change in hepatosteatotic state are lacking.

Methods 394 participants, aged 63-79 years, from the Edinburgh Type 2 Diabetes Study, a large, randomly-selected population of patients with Type 2 diabetes, were assessed on two occasions approximately 3 years apart. Liver ultrasonography was undertaken and both plasma alanine aminotransferase (ALT) and aspartateaminotransferase (AST)) were measured. Hepatic steatosis was graded as either "normal", "mild" or "moderate/severe" according to findings on ultrasound. Change in steatosis was classified as either regression or progression by 1 or 2 categories. ANOVA (with trend) analysis was used to assess the association between change in steatosis and change in aminotransferase levels.

Results Mean follow-up was 2.7 years. $5.1 \%(n=20)$ of participants regressed two categories, $14.7 \% \quad(n=58)$ regressed one category, $60.7 \%(n=239)$ showed no change, $12.4 \%(n=49)$ progressed one category and $7.1 \%(n=28)$ progressed two categories. There was a statistically significant linear trend for both ALT and AST $(F(4,3)=$ $4.76, \mathrm{p}=0.03$ and $F(4,3)=10.70, \mathrm{p}=0.01$ respectively) indicating that as the hepatosteatosis stage changed the aminotransferase level increased or decreased proportionally.

Conclusion In a relatively large sample of patients with type 2 diabetes representative of patients with type 2 diabetes in general, we have shown that change in aminotransferase levels are proportionally associated with change in levels of hepatosteatosis. This suggests that aminotransferases may be useful in monitoring progression of steatosis.

\section{P2-202 THIAZOLIDINEDIONES ARE ASSOCIATED WITH REGRESSION OF HEPATOSTEATOSIS IN PEOPLE WITH TYPE 2 DIABETES: THE EDINBURGH TYPE 2 DIABETES STUDY}

doi:10.1136/jech.2011.142976j.35

${ }^{1} \mathrm{~J}$ Morling, ${ }^{*}{ }^{2} \mathrm{R}$ Williamson, ${ }^{2} \mathrm{M}$ Strachan, ${ }^{1} \mathrm{~J}$ Price, ${ }^{2} \mathrm{~L}$ Nee, ${ }^{2} \mathrm{~S}$ Glancy. ${ }^{1}$ University of Edinburgh, Edinburgh, UK; ${ }^{2}$ Western General Hospital, Edinburgh, UK

Background Increasing evidence suggests that some antidiabetic medications may improve markers of abnormal liver structure and function in people with non-alcoholic fatty liver disease. We investigated this association in older people with type 2 diabetes.

Methods 474 participants, aged 63-79 years, from the Edinburgh Type 2 Diabetes Study, a large, randomly-selected population of patients with Type 2 diabetes, underwent assessment on two occasions. At baseline, liver ultrasonography was undertaken and antidiabetic medications (metformin, sulphonyureas and thiazolidinediones) were recorded. Liver ultrasonography was repeated approximately 3 years later. Hepatosteatosis was graded as either normal or "fatty", and the change between examinations as regression, no change or progression. $\chi^{2}$ for trend was used to analyse the association.

Results Mean follow-up was 2.7 years. $9.5 \%(n=45)$ of participants progressed, $13.9 \%(n=66)$ regressed and $76.6 \%(n=363)$ remained the same. Thiazolidinedione use was significantly higher among participants whose hepatosteatosis regressed $(5.0 \%, 11 \%$ and $19.7 \%$ in participants with progression, no change and regression respectively, $\mathrm{p}=0.02$ ). Similar figures for metformin use were $55.0 \%, 67.0 \%$ and $67.2 \%$ respectively $(p=0.28)$ and for sulphonyurea use were $15.0 \%, 34.9 \%$ and $31.1 \%$ respectively $(p=0.19)$.

Conclusion In a large sample of patients with type 2 diabetes representative of patients with type 2 diabetes in general, thiazolidinedione use was associated with regression of hepatosteatosis. This is consistent with emerging evidence from clinical trials suggesting that there may be a role for thiazolidinediones beyond glucose control in patients with type 2 diabetes. Further analyses are warranted to explore the potential mechanism underlying this association.

\section{P2-203 ASSOCIATION OF VITAMIN D AND CARDIOMETABOLIC RISK FACTORS AMONG A MALAY COHORT IN KUALA LUMPUR, MALAYSIA}

doi:10.1136/jech.2011.142976j.36

${ }^{1,2} \mathrm{~F}$ M Moy. ${ }^{1}$ Faculty of Medicine, University of Malaya, Kuala Lumpur, Malaysia; ${ }^{2}$ Julius Centre University of Malaya, Kuala Lumpur, Malaysia

Introduction Epidemiologic studies suggest that vitamin D (25hydroxyvitamin D) is inversely associated with metabolic syndrome in the western populations. However, evidence from Asian population is limited. The present study was conducted to investigate the association of vitamin $\mathrm{D}$ and cardiometabolic risk factors among an existing Malay cohort in Kuala Lumpur.

Methods This is an analytical cross sectional study. A total of 380 subjects were sampled. Their vitamin D status, fasting blood glucose, full lipid profile, blood pressure, weight, height and waist circumference were measured.

Results There were more (58\%) female respondents. Their mean age was $48.5 \pm 5.2$ years. The prevalence of Metabolic Syndrome was $37.0 \%$ while the mean vitamin D level was 44.5 (95\% CI 42.6 to 46.4) $\mathrm{nmol} / \mathrm{l}$. Females had significantly lower mean vitamin $\mathrm{D}$ level (36.3; 95\% CI 34.5 to $38.0 \mathrm{nmol} / \mathrm{l})$ compared to males $(56.1 ; 95 \% \mathrm{CI}$ 53.2 to $59.2 \mathrm{nmol} / \mathrm{l}$ ). Respondents with low (cut off at $50 \mathrm{nmol} / \mathrm{l}$ ) vitamin D level had 2.63 (95\% CI 1.58 to 4.36) times odds of having abdominal obesity. Low vitamin $\mathrm{D}$ levels were associated with higher odds of low HDL-lipoprotein (OR: 1.26; 95\% CI 0.70 to 2.27), high fasting blood glucose (OR: $1.22 ; 95 \%$ CI 0.70 to 2.12 ), abnormal/high triglyceride (OR: 1.46 ; $95 \%$ CI 0.87 to 2.47 ) and abnormal systolic and/or diastolic blood pressure (OR: 1.43; 95\% CI 0.85 to 2.38). Respondents with lower vitamin D levels had higher odds for Metabolic Syndrome (OR: 1.70; 95\% CI 1.01 to 2.89).

Conclusion Our results concur with those from the West where vitamin $\mathrm{D}$ deficiencies are associated with cardiometabolic risk factors.

\section{P2-204 ASSOCIATION OF BODY MASS INDEX AND HEALTH- RELATED QUALITY OF LIFE IN HIGH-RISK CARDIOVASCULAR PATIENTS}

doi:10.1136/jech.2011.142976j.37

\begin{abstract}
${ }^{1,2} \mathrm{~J}$ Müller-Nordhorn, ${ }^{3} \mathrm{H}$ Englert, ${ }^{2} \mathrm{R}$ Muckelbauer, ${ }^{4} \mathrm{~K}$ Wegscheider, ${ }^{5} \mathrm{H}$ Völler, ${ }^{6}$ F Sonntag, ${ }^{7} \mathrm{H}$ A Katus, ${ }^{1} \mathrm{~S} N$ Willich. ${ }^{1}$ Institute for Social Medicine, Epidemiology and Health Economics, Berlin, Germany; ${ }^{2}$ Berlin School of Public Health, Berlin, Germany; ${ }^{3}$ University of Applied Sciences, Münster, Germany; ${ }^{4}$ Department of Medical Biometry and Epidemiology, University of Hamburg, Hamburg, Germany; ${ }^{5}$ Rehabilitation Center for Cardiovascular Diseases, Rüdersdorf, Germany; ${ }^{6}$ Cardiology Practice, HenstedtUlzburg, Germany; 'Department of Cardiology, University of Heidelberg, Heidelberg, Germany
\end{abstract}

Introduction Apart from clinical events, health-related quality (HRQoL) is an important outcome in high-risk cardiovascular patients. As body mass index has been associated with clinical events ("obesity paradox"), we investigated the relationship between body mass index and health-related quality of life.

Methods Patients were included with hypercholesterolaemia and an indication for statin therapy in 1961 primary care practices. HRQoL was assessed with the Short Form (SF)-12 health status instrument at baseline, after 6 and after 12 months. Physicians assessed patient body mass index (BMI). A mixed-effects regression model accounting for the three measurement points was used to investigate (a) the association between BMI and HRQoL at baseline, and (b) the association between change in BMI and change in HRQoL. Results A total of 5082 patients (2165 females) were included. The mean change in BMI within 12 months was 0.05 (SD: 1.11) kg/m². 
At baseline, BMI was inversely associated with physical and mental SF-12 summary scores $(\beta[95 \% \mathrm{CI}]=-0.3[-0.4$ to -0.2$]$, and -0.1 [-0.1 to 0.0]). An increase in BMI of $1 \mathrm{~kg} / \mathrm{m}^{2}$ within 12 months was associated with a significant decrease in physical but not mental SF12 scores $(0.3[-0.4$ to -0.1$])$. Due to an interaction with gender, the association between change in BMI and physical SF-12 scores was only prevalent in women $(\beta=-0.5[-0.7$ to -0.2$], p<0.001)$ but not in men $(\mathrm{p}=0.32)$.

Conclusion BMI is inversely associated with quality of life in highrisk cardiovascular patients. Increases in BMI may lead to a decrease in physical quality of life in women but not in men.

\section{P2-205 HETEROGENEITY IN FUNCTIONAL RECOVERY AFTER STROKE: AN EXPLORATORY STUDY USING LONGITUDINAL LATENT CLASS ANALYSIS}

doi:10.1136/jech.2011.142976j.38

T Munyombwe, ${ }^{*}$ R West, K Hill, Y-K Tu, P Knapp. University of Leeds, Leeds, UK

Introduction Prediction models for functional recovery after stroke can be improved by adjusting for the heterogeneity in functional recovery patterns. This study explored the heterogeneity in functional recovery after stroke using longitudinal latent class analysis and characterised the patients in the different latent classes.

Methods The analyses were performed on a data set from a cohort of 448 stroke survivors participating in a study of outcomes at 1 year. Heterogeneity in functional recovery after stroke was investigated using Longitudinal Latent class analysis of total Barthel scores measured at 1, 6 and 12 months after stroke. Identification of the optimal number of classes was based on BIC, AIC, and LoMendell-Rubin Adjusted Likelihood ratio test. The second analysis characterised the latent classes.

Results A four latent class structure was preferred. All the four latent classes showed a non linear pattern of recovery over time. Persons in the very poor functional recovery group had the largest median length of initial hospital stay 99 (13-257 days), mean age $75 \pm 9.27$ years and greatest probability of being urinary and bladder incontinence. The group with best functional recovery had the least initial hospital stay 14 (2-147 days), least proportion of people with previous stroke, least proportion of people with urinary and bladder incontinence, the mean age at admission was $68.76 \pm 11.72$ years.

Conclusion The study showed that there is heterogeneity in functional recovery patterns after stroke. Latent class analysis is a useful method for identifying subgroups of functional recovery after stroke.

\section{P2-206 ASSOCIATION OF FAAR SCORE ON ADMISSION ECG WITH MORTALITY IN 1843 PATIENTS ADMITTED WITH AN ACUTE CORONARY SYNDROME}

doi:10.1136/jech.2011.142976j.39

\begin{abstract}
${ }^{1} \mathrm{M}$ Lown, ${ }^{2} \mathrm{~T}$ Munyombwe, ${ }^{*} \mathrm{~W}$ Harrison, ${ }^{2} \mathrm{R}$ West, ${ }^{1} \mathrm{C}$ Hall, ${ }^{1} \mathrm{C}$ Morrell, ${ }^{1} \mathrm{~B}$ Jackson, ${ }^{1} \mathrm{R}$ Sapsford, ${ }^{1} \mathrm{~N}$ Kilcullen, ${ }^{1} \mathrm{C}$ Pepper, ${ }^{3} \mathrm{P}$ Batin, ${ }^{1} \mathrm{~A}$ Hall, ${ }^{1,2} \mathrm{C}$ Gale, ${ }^{5} \mathrm{~A}$ Simms. ${ }^{1}$ University of Leeds, Center for Epidemiology and Biostatistics, Leeds, UK; ${ }^{2}$ Department of Cardiology, Leeds Teaching Hospitals NHS Trust, Leeds, UK; ${ }^{3}$ Department of Cardiology, Mid-Yorkshire NHS Trust, Wakefield, Wakefield, UK; ${ }^{4}$ Department of Cardiology, York Hospitals NHS Foundation Trust, York, UK; ${ }^{5}$ Leeds General Infirmary, Leeds, UK
\end{abstract}

Introduction Accurate risk assessment is central to the management of patients presenting with acute coronary syndromes (ACS). Many ACS risk scores are employed as near-point tests, but rely on the collection of remote data such as troponin concentration. This study investigated the long term and short term mortality prediction performance of the Frontal ORS-T Angle and Age Risk (FAAR) score, a simple ACS risk stratification tool comprising of the frontal ORS$T$ angle and age at admission and compared with GRACE risk score which relies on a multitude of clinical variables.

Method Data from the Evaluation of Methods and Management of Acute Coronary Events EMMACE-2 and EMMACE-1 prospective studies was used to test and validate models respectively. EMMACE studies examined outcomes in consecutively admitted, unselected patients with confirmed ACS in multiple adjacent hospitals (within the catchment area of one tertiary centre) in Yorkshire, UK. Using the EMMACE-2 (2499 patients), FAAR score adjusted for patient characteristics, a stepwise logistic regression and proportional hazards Cox regression models was used to predict 30-day and 2 year mortality.

Results The FAAR score offered excellent discriminative performance for 30-day, C statistic (95\% CI) 0.74 (0.71 to 0.78) and 2-year 0.76 (0.74 to 0.78 ) mortality, maintained its performance in the EMMACE-1 validation cohort at 30-days, C statistic (95\% CI) 0.76 (0.71 to 0.80$)$ and at 2 -years 0.79 (0.75 to 0.83 ), and compared favourably with the GRACE score.

Conclusion FAAR score could be used by emergency healthcare professionals to assist the triage of patients presenting with suspected ACS.

\section{P2-207 EPIDEMIOLOGY OF NON-COMMUNICABLE DISEASES AND THEIR RISK FACTORS IN RURAL UGANDA}

doi:10.1136/jech.2011.142976j.40

${ }^{1} \mathrm{G}$ Murphy, ${ }^{* 2} \mathrm{R}$ Nsubuga, ${ }^{2} \mathrm{G}$ Asiki, ${ }^{2,4} \mathrm{~J}$ Seeley, ${ }^{1,3} \mathrm{E}$ Young, ${ }^{2,5} \mathrm{~A}$ Kamali. ${ }^{1}$ University of Cambridge, Cambridge, UK; ${ }^{2}$ MRC/UVRI Uganda Research Unit on AIDS, Entebbe, Uganda; ${ }^{3}$ Wellcome Trust Sanger Institute, Hinxton, UK; ${ }^{4}$ University of East Anglia, Norwich, UK; ${ }^{5}$ London School of Hygiene and Tropical Medicine, London, UK

Introduction Non-communicable diseases (NCDs) are rapidly becoming leading causes of morbidity and death in low- and middleincome countries, including those in sub-Saharan Africa. Yet, the magnitude and distribution of established and emerging risk factors for NCDs have not been fully studied in sub-Saharan African countries in a large scale epidemiological context.

Methods We will carry out a cross-sectional population-based survey of cardiometabolic risk factors and disease of approximately 8000 participants ages 13 years and older, in a rural population in Uganda. Trained field staff will conduct a questionnaire based upon the WHO STEPwise approach to Surveillance questionnaire; perform biophysical measurement, including anthropometry and blood pressure readings; and collect blood samples for biochemical analysis for cardiometabolic risk factors and infection.

Results By August 2011, data on approximately 6000 participants will have been collected. We will report initial findings, including the prevalence and distribution of lifestyle risk factors, physical measurements and biochemical measurements. Lifestyle risk factors will include tobacco use, alcohol consumption, diet and physical activity. Physical measurements will include blood pressure, body mass index and waist hip ratio, as indicators of hypertension and obesity, respectively. Biochemical measurements will include liver function tests, lipid levels and HbAlc as an indication of diabetes. Age and sex specific estimates will be reported.

Conclusion Population based epidemiological studies can provide reliable data on disease burden and their risk factors to help inform public health policy and programmes aimed at addressing the rise in NCDs in Uganda. 